\title{
Impact of the Supporting Physical Activity in the Childcare Environment (SPACE) intervention on preschoolers' physical activity levels and sedentary time: a single- blind cluster randomized controlled trial
}

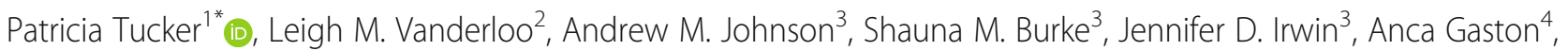
Molly Driediger ${ }^{1}$ and Brian W. Timmons ${ }^{5}$

\begin{abstract}
Background: Physical activity levels among preschoolers in childcare are low and sedentary time high. The Supporting Physical Activity in the Childcare Environment (SPACE) intervention had three components: 1. portable play equipment; 2. staff training; and, 3. modified outdoor playtime (i.e., shorter, more frequent periods). This study aimed to examine the effectiveness of the SPACE intervention on preschoolers' physical activity levels and sedentary time during childcare hours (compared to standard care).

Methods: Via a single-blind cluster randomized controlled trial, 338 preschoolers (39.86 \pm 7.33 months; 52\% boys) from 22 centre-based childcare facilities (11 experimental, 11 control) were enrolled. Preschoolers wore an Actical ${ }^{\mathrm{TM}}$ accelerometer for 5 days during childcare hours at baseline, post-intervention, and 6- and 12-month follow-up, and were included in the analyses if they had a minimum of two valid days ( $5 \mathrm{~h}$ each day) at baseline and one additional time point. Intervention effectiveness was tested using a linear mixed effects model for each of the four outcome variables (i.e., sedentary time, light physical activity [LPA], moderate-to-vigorous physical activity [MVPA], and total physical activity [TPA]). Fixed effects were further evaluated with $t$-tests, for which degrees of freedom were estimated using a Satterthwaite approximation.

Results: One hundred and ninety-five preschoolers were retained for analyses. The intervention did not significantly impact LPA. MVPA was significantly greater among children in the experimental group when comparing postintervention to pre-intervention, $t(318)=3.50, p=.0005$, but no intervention effects were evident at 6 - or 12-month follow-up. TPA was significantly greater for children in the intervention group at post-intervention when compared to pre-intervention, $t(321)=2.70, p=.007$, with no intervention effects evident at later time periods. Finally, sedentary time was significantly lower among preschoolers in the experimental group when comparing postintervention to pre-intervention, $t(322)=2.63, p=.009$, with no significant effects at follow-up.

(Continued on next page)
\end{abstract}

\footnotetext{
* Correspondence: ttucker2@uwo.ca

${ }^{1}$ School of Occupational Therapy, Faculty of Health Sciences, University of Western Ontario, 1201 Western Road, Elborn College, Room 2547, London, ON N6G 1H1, Canada

Full list of author information is available at the end of the article
} International License (http://creativecommons.org/licenses/by/4.0/), which permits unrestricted use, distribution, and reproduction in any medium, provided you give appropriate credit to the original author(s) and the source, provide a link to the Creative Commons license, and indicate if changes were made. The Creative Commons Public Domain Dedication waiver (http://creativecommons.org/publicdomain/zero/1.0/) applies to the data made available in this article, unless otherwise stated. 
(Continued from previous page)

Conclusions: The SPACE intervention was effective at increasing MVPA and TPA among preschoolers, while simultaneously decreasing sedentary time. The ability of the SPACE intervention to target higher intensity activity is promising, as MVPA levels have been documented to be low in centre-based childcare. The changes in physical activity were not sustained long term (6- or 12-month follow-up).

Trial registration: ISRCTN70604107 (October 8, 2014).

Keywords: Physical activity, Sedentary time, Childcare, Preschooler, Outdoor playtime, Early years, Intervention

\section{Background}

Physical activity participation among preschoolers (defined herein as $2.5-5$ years) in centre-based childcare facilities has been consistently reported as low [1-4]; likewise, the prevalence of sedentary time has been noted as high in this environment [5-7]. In fact, previous research contends that preschoolers in childcare centres spend only $1.5 \mathrm{~min} / \mathrm{h}$ in moderate-to-vigorous physical activity (MVPA), devoting the majority of their time (40.6 $\mathrm{min} / \mathrm{h})$ to sedentary pursuits [1]. Unfortunately, these trends could have potentially devastating impacts on the health and development of young children. Physical activity has been identified as positively impacting cardiovascular health, and is associated with improved weight status and better psychosocial and cognitive development $[8,9]$. Similarly, excess sedentary time has been linked with a risk for increased adiposity and poorer psychosocial health and cognitive development [10, 11]. Canadian Physical Activity Guidelines recommend the accumulation of 180 min of physical activity (at any intensity) per day among this young population [12], moving towards $60 \mathrm{~min}$ of MVPA by the age of 5 years. Canada's Sedentary Behaviour guidelines (for children aged 2-4 years), encourage minimizing sedentary pursuits for overall healthy growth and development, and specifically advise against more than one hour of daily screen time [13]. As such, early intervention is needed to support the development of appropriate physical activity and sedentary levels among preschoolers.

Typified by providing care to young children in a classroom-like setting, centre-based childcare has received notable attention in the literature, particularly regarding obesity-related behaviours (such as physical activity and screen-viewing) [14, 15]. Often recognized as an obesogenic and sedentary environment $[2,6,16]$, researchers suggests that the childcare environment accounts for roughly $50 \%$ of the variation in preschoolers' physical activity $[3,17]$. More specifically, a number of childcare factors, including portable play equipment and supportive staff behaviours have been recognized as strong correlates to physical activity participation [2]. The majority of Canadian preschoolers attend some form of non-parental care $[18,19]$. This fact, combined with previous findings that those who are cared for in this particular environment (compared to those cared for by a parent or relative) are at a higher risk for becoming obese in later childhood [20], make centre-based childcare facilities an appropriate, if not necessary, venue for targeting health promotion efforts.

Evidence-informed action is needed to improve and support the physical activity levels and reduce the sedentary time of preschoolers in childcare. Recently, steps have been taken to understand the effective qualities of physical activity interventions targeting this population. Via systematic review and meta-analysis, Ward et al. [21] and Gordon et al. [22], both highlighted the effectiveness of intervening in childcare centres and offering environmental modifications (e.g., portable play equipment, floor markings) as successful approaches to improving young children's physical activity levels. Moreover, the importance of outdoor play was also supported [22]. This is no surprise given that, generally, young children exhibit higher levels of physical activity when outdoors [23-27]. This sentiment is echoed by the 2015 ParticipACTION Position Statement on Outdoor Active Play which highlighted the physiological and psychosocial benefits gained by children playing outside [28]. Specific to the childcare environment, a recent study by Vanderloo et al. [27] reported that preschoolers accumulated significantly higher rates of physical activity and significantly lower sedentary time during outdoor play periods than when indoors at childcare. Moreover, preschoolers' activity levels have been shown to be highest when they are first exposed to their outdoor environment (i.e., within the first $10 \mathrm{~min}$ ), with activity levels declining with increased duration of outdoor play [29,30]. Given the positive impact of outdoor playtime on this population's activity levels, interventions adopting this approach show great promise.

At an international level, several interventions have recently been undertaken in the childcare setting [31-40]. These studies underscore not only the appropriateness of the childcare venue for intervention, but also the pressing need to improve activity behaviours of young children enrolled in these settings. With consolidated evidence now available regarding effective strategies for improving preschoolers' physical activity levels [21,22], and with consideration of childcare providers' perspectives regarding the 
barriers and facilitators to engaging preschoolers in physical activity in childcare [41, 42], the 8-week Supporting Physical Activity in the Childcare Environment (SPACE) intervention was created. The SPACE intervention was developed primarily to support preschoolers' physical activity, and secondarily to decrease sedentary time, in centre-based childcare via a unique combination of environmental modifications, staff training, and modified outdoor playtime periods.

The purpose of this randomized controlled trial (RCT) was to examine the effectiveness of the SPACE intervention with regard to increasing preschoolers' physical activity levels and decreasing sedentary time during childcare hours. It was hypothesized that preschoolers assigned to the experimental condition would display higher rates of physical activity and lower rates of sedentary time compared to preschoolers in the control condition, from preto post-intervention, and at 6- and 12-month follow-up periods.

\section{Methods \\ Description of SPACE intervention}

The SPACE study was an evidence-informed physical activity intervention comprised of the following components: 1. environmental modifications (i.e., provision of novel portable play equipment including balls, hula hoops, a hop-scotch mat, obstacle course, stepping domes, ribbon wands, and hop-along bouncers); 2. staff training (i.e., one 4-h physical activity training session for staff and directors that emphasized the importance of shorter bouts of physical activity and reduced sedentary time, recommendations for overcoming obstacles, explanation of the Canadian physical activity and sedentary behaviour guidelines, and provided examples of activities that could be implemented in childcare); and, 3. modified outdoor playtime (i.e., re-structuring the provincially required two 60 -min outdoor sessions into four 30-min periods). Given recent evidence to suggest that preschoolers' activity levels are highest during their first 10 min outdoors, and consequently, that simply extending outdoor playtime may not be adequate for promoting physical activity [29], the SPACE intervention consisted of a modified outdoor playtime schedule to increase the frequency, but not the duration, of unstructured outdoor playtime provided to young children in childcare. See Tucker et al. [43] for additional details regarding each component of the intervention and supporting evidence.

\section{Study design and recruitment}

A detailed methodological account has been published elsewhere [43]. Informed by the PRECEDE-PROCEED model for health promotion program planning [44], and methodologically designed and implemented in accordance with the Consolidated Standards of Reporting
Trials (CONSORT) statement [45], a single-blind cluster RCT was conducted. Childcare centres were eligible if they had at least one preschool classroom, if the staff and children were English-speaking, and if the centre director and childcare staff of the eligible classrooms were willing to participate. All recruitment and randomization activities were conducted by the project coordinator. Twenty-two centre-based childcare facilities in London, Ontario, Canada were randomly selected and agreed to participate (59\%; see Fig. 1 for participation rates). The director of each childcare facility was contacted via phone; once verbal consent was received from all 22 centres, each centre was randomly assigned to either the experimental or control condition (using a blocked design). Centre start dates were staggered over four months during spring and summer, making it logistically impossible to perform baseline measures prior to randomization. No centres withdrew from the study at any time. Only centres assigned to the experimental condition had childcare staff deliver the SPACE intervention (i.e., providing four 30-min daily outdoor periods and offering the supplied portable play equipment), while centres enrolled in the control condition continued their typical daily curriculum and programming, including regular outdoor playtime periods (i.e., two 60-min periods). This study and all related documents received ethical approval from the University of Western Ontario's Research Ethics Board (REB\# 105779) and was assigned an International Standard Randomized Controlled Trial Number (ISRCTN 70604107).

\section{Participants}

Parents/guardians of typically developing children (i.e., free of any chronic disease or developmental issue) within the preschool classroom(s) of the selected childcare centres were invited to participate. Preschoolers were eligible if they were between the ages of 2.5 and 4 years and had a parent/guardian who spoke and understood English to provide informed consent. Based on the power calculation conducted for the SPACE study [43], the recruitment goal was 348 preschoolers from 22 childcare centres.

\section{Data collection}

Data collection occurred between March 2015 and October 2016, with the intervention being implemented, in a staggered format, from April to July 2015 (see Fig. 2 for SPACE timeline). Measurements were completed for preschoolers in both the experimental and control condition at baseline (i.e., week 0 ), immediately postintervention (i.e., week 8), and at 6- and 12-month follow-up. Trained in conducting anthropometric measurements (inter-rater reliability, $r=.99$ ), research assistants $(n=2)$, who were blind to group assignment, completed all measurements. Research staff visited 


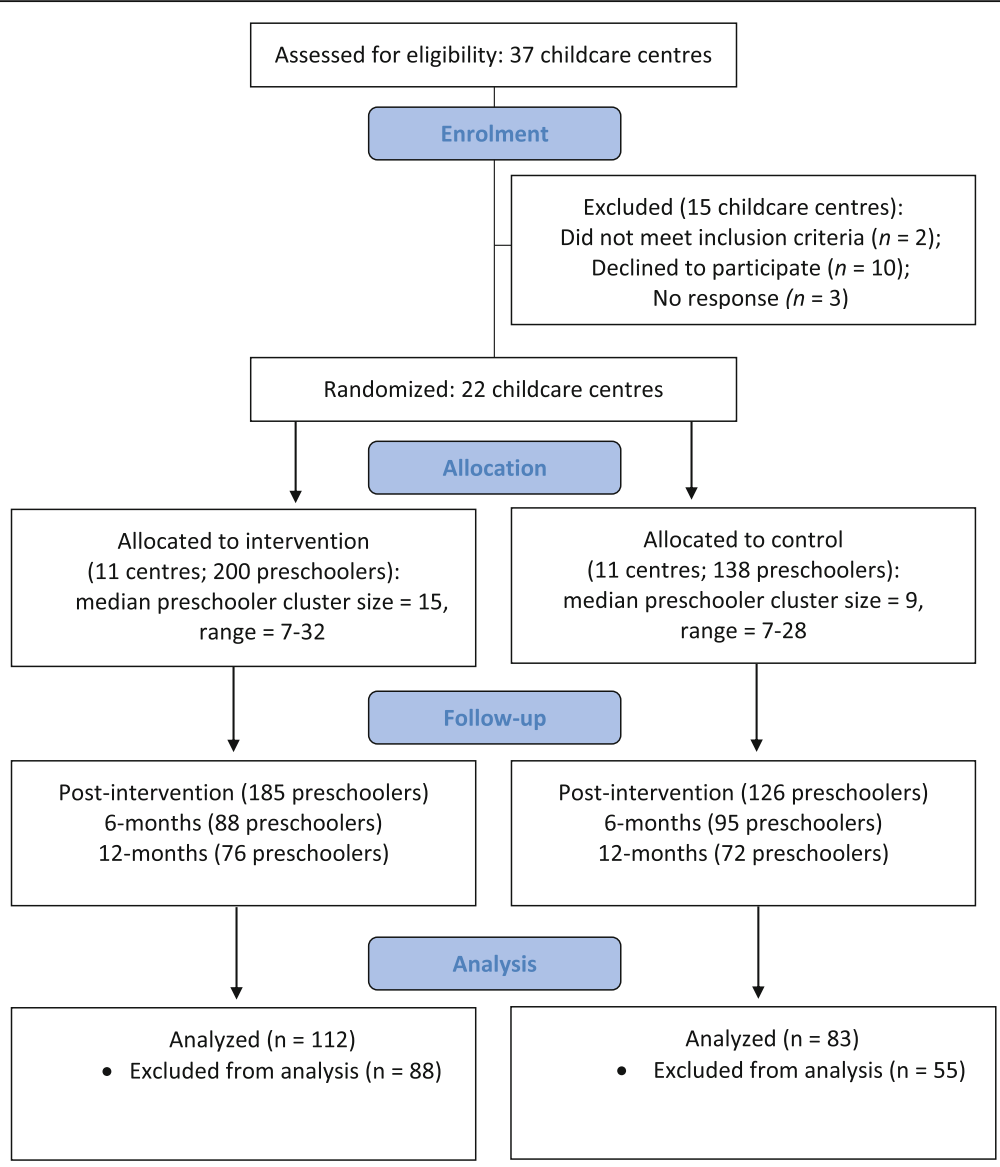

Note. No centres were lost to follow-up or dropped out of the intervention. Preschoolers were excluded due to: Inadequate wear-time, absence during data collection period, withdrawal from childcare, lost device, and device malfunction.

Fig. 1 CONSORT flow diagram for the SPACE intervention

participating childcare centres prior to the onset of each data collection period to distribute the accelerometers (including instruction sheets and daily wear-time logs) and questionnaire packages.

\section{Tools}

Accepted as a valid and reliable tool for assessing young children's activity behaviours [46, 47], Actical $^{\mathrm{TM}}$ accelerometers
( $Z$ and B series; Phillips Respironics, Bend, Oregon) were used to objectively measure participants' physical activity and sedentary time. Worn on participants' right hip and secured with an elastic waist band, preschoolers were asked to wear the accelerometer for five consecutive days, during childcare hours only, at each of the four time points. Childcare staff were instructed to place the devices on the children's right hip when they arrived at the centre

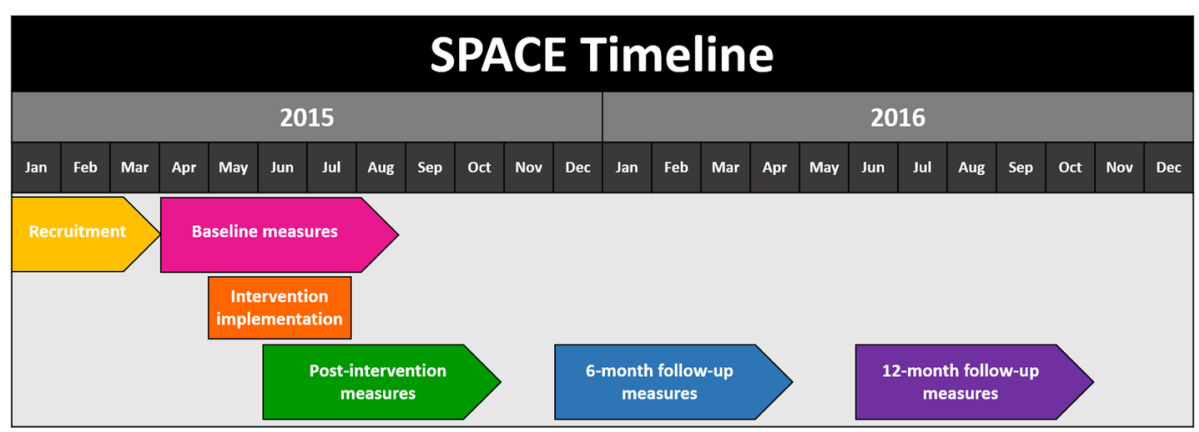

Fig. 2 The Supporting Physical Activity in the Childcare Environment (SPACE) Intervention Timeline 
and to remove them at end-of-day prior to leaving for home. Staff were also asked to keep a daily wear-time log for each participating child.

Accelerometers were programmed using a 15-s epoch length, and in line with the Canadian Health Measures Survey [48], cut-points (all divided by four to match the time sampling interval used in the present study) by Adolph and colleagues [49] were used: light physical activity, LPA: $\geq 25 \leq 287.25$ counts $\cdot 15 \mathrm{~s}^{-1}$.epoch ${ }^{-1}$; MVPA $\geq 287.5$ counts $15 \mathrm{~s}^{-1} \cdot$ epoch $^{-1}$; total physical activity, TPA: $\geq 25$ counts $15 \mathrm{~s}^{-1}$.epoch ${ }^{-1}$. Sedentary cut-points [50] were also applied to the collected accelerometer data: $\leq 24.75$ counts $\cdot 15 \mathrm{~s}^{-1}$. Rates $(\mathrm{min} / \mathrm{h})$ were calculated to account for differences across groups (i.e., varying wear-times due to differences in childcare hours and attendance).

Prior to each data collection time-point, anthropometric measurements were taken at the childcare centre to calculate preschoolers' body mass index (BMI) percentiles. Height was measured to the nearest $0.1 \mathrm{~cm}$ using a Seca 214 "Road Rod" Portable Stadiometer, weight was measured to the nearest $0.1 \mathrm{~kg}$ using a Tanita 700-TBF300GS Body Fat Analyzer w/Goal Setter scale, and waist circumference was measured to the nearest $0.1 \mathrm{~cm}$ at the child's naval using an anthropometric measuring tape. Prior to these measurements, participants were asked to remove their shoes and any heavy clothing.

A demographic questionnaire was also administered to the parents/guardians of participating children. This questionnaire was completed at baseline only and was used to collect information on potential correlates of participants' activity levels. Such items included: age, sex, and ethnicity of preschooler; yearly family income; parent/guardian education level; family status, and child's participation in extra-curricular activities outside of childcare hours.

\section{Data analysis}

To compare demographic variables between the experimental and control groups, continuous variables were evaluated using independent sample $t$-tests, while categorical variables were explored using Pearson chi-square calculations.

Accelerometer data were downloaded using device specific software (Actical ${ }^{\mathrm{Tm}}$ version 3.10) and analyzed at an epoch length of $15 \mathrm{~s}$. Files were then uploaded to KineSoft (version 3.3.62; Loughborough, United Kingdom) to assess the quality of the data files for each participant at each time point. Only participants with a minimum of 2 valid days (where $5 \mathrm{~h}$ of wear-time equated to a valid day) at baseline and one additional time point, were retained for analyses. Non-wear time was defined as $20 \mathrm{~min}$ of consecutive zeros [51, 52]. See Table 1 for additional details regarding accelerometer data collection and compliance.

LPA, MVPA, TPA, and sedentary time were the outcomes of interest for the present study. Each outcome variable was evaluated within a linear mixed effects model, with group (experimental versus control) and time (baseline, post-intervention, 6-month follow-up, and 12-month follow-up) as fixed effects. This analytic approach reduces concerns regarding missing data, as participant error is modeled within the design as a random effect. This approach also allows for the testing of cluster effects (i.e., the childcare centre from which the individual was recruited), to ensure that the randomization method did not have a systematic effect on the outcome. To identify the model of best fit, the following models were tested: 1 . a "null model"; 2. a "main effects only" model (i.e., a model in which group and time were not allowed to correlate); 3. an "interaction" model (i.e., a model in which group and time were allowed to correlate); and, 4 . a model that considered cluster effects. Models 1 through 3 were tested hierarchically (i.e., Model 2 was compared with Model 1, and Model 3 was compared with Model 2). Model 4 (the model that included cluster effects) was, for each dependent variable, compared against the best fitting model that did not include cluster effects. In each case, the model comparison was conducted using a Pearson chi-square test. With the reporting of the model of best fit, parameters were compared to the reference group using a $t$-test, and degrees of freedom were estimated with a Satterthwaite approximation [53]. All models were fitted using the lme4 package [54] in R [55], and the $t$-tests used in the interpretation of model effects were computed using the ImerTest package [56].

\section{Results \\ Description of sample}

A total of 338 preschoolers (39.86 \pm 7.33 months; $52 \%$ boys) enrolled in the SPACE study (response rate of 73\%). Children in the treatment group $(M=40.61, S D=7.31)$ were significantly older than children in the control group $(M=38.72, S D=7.24), t(307)=2.23, p=0.026$. There

Table 1 Accelerometer compliance for the SPACE Study

\begin{tabular}{lllll}
\hline & Pre-Intervention & Post-Intervention & 6-Month Follow-Up & 12-Month Follow-Up \\
\hline Number of Devices Distributed & 335 & 313 & 180 & 141 \\
Number of Devices Not Returned & 1 & - & - & 1 \\
Number of Devices that Malfunctioned & 4 & - & 2 & - \\
\hline
\end{tabular}


was no significant difference in BMI percentile between the treatment group $(M=58.21, S D=27.78)$ and the control group $(M=56.03, S D=29.69), t(247)=.59$, $p=0.56$. When our accelerometer wear time parameters were applied ( 2 valid days), 195 participants were retained for analyses. Age (months) differed significantly between compliant $(M=40.53 ; S D=7.76)$ and non-compliant $(M=38.93 ; S D=6.6)$ participants $t(298)=1.95$, $p=0.052$; however, no other significant differences in baseline characteristics were found between children with incomplete or complete baseline PA data in either group. See Table 2 for full demographic information of the participating preschoolers.

\section{Effects of the SPACE intervention on physical activity and sedentary time}

Means and standard deviations for physical activity intensities and sedentary time, separated by time and group, are presented in Table 3. Rates of MVPA, TPA, and sedentary time are displayed in Fig. 3 . The results of the linear mixed-effects model-testing is presented in Table 4. Cluster effects were non-significant for all four outcome variables.

The results presented in Table 4 suggest that the intervention was effective at improving preschoolers' MVPA and TPA, and decreasing sedentary time, indicated by a statistically significant interaction between group (i.e., experimental vs. control) and time (i.e., baseline and post-intervention only). For all three of these variables, the experimental group displayed a significant difference between baseline and post-intervention as compared to the control group, but no significant effects were found at 6- or 12-month follow-up. Both MVPA, $t(317)=3.47$ $(p=0.0005)$, and TPA, $t(321)=2.70(p=0.007)$, were significantly higher post-intervention, while sedentary time was significantly less post-intervention, $t(322)=2.632$ $(p=0.009)$.

\section{Discussion}

The purpose of this study was to examine the impact of the SPACE intervention on the physical activity levels and sedentary time of preschoolers enrolled in centrebased childcare. The SPACE study is unique because it entails a combination of evidence-informed components known to be associated with preschoolers' physical activity and sedentary time while in childcare. The results of this study suggest that the 8-week SPACE intervention was effective at increasing preschoolers' MVPA and TPA levels and at decreasing sedentary time from pre- to post-intervention. However, changes to activity behaviours and sedentary time were not sustained (at 6- or 12-month follow-up) when the intervention was removed.
Table 2 Descriptive characteristics of enrolled preschoolers

\begin{tabular}{|c|c|c|c|}
\hline Variable & Control & Intervention & $p$-value \\
\hline Age (months), $M(S D)$ & $38.72(7.24)$ & $40.61(7.31)$ & .03 \\
\hline Sex (male/female), $n$ & $76 / 62$ & $102 / 98$ & .46 \\
\hline BMI Percentiles, M (SD) & $56.03(29.69)$ & $58.21(27.78)$ & .26 \\
\hline Hours in Childcare (hours) & & & .82 \\
\hline$<10$ & 3 & 4 & \\
\hline $10-19$ & 8 & 18 & \\
\hline $20-29$ & 16 & 23 & \\
\hline $30+$ & 95 & 142 & \\
\hline Ethnicity & & & .48 \\
\hline Caucasian & 87 & 142 & \\
\hline African Canadian & 1 & 4 & \\
\hline Aboriginal & 5 & 4 & \\
\hline Arab & 2 & 3 & \\
\hline Latin-American & 3 & 6 & \\
\hline Asian & 7 & 7 & \\
\hline Other & 19 & 19 & \\
\hline Family Income & & & .65 \\
\hline$<\$ 20,000$ & 11 & 9 & \\
\hline$\$ 20,000-\$ 39,999$ & 13 & 14 & \\
\hline$\$ 40,000-\$ 59,999$ & 10 & 14 & \\
\hline$\$ 60,000-\$ 79,999$ & 12 & 11 & \\
\hline$\$ 80,000-\$ 99,999$ & 8 & 16 & \\
\hline$\$ 100,000-\$ 119,999$ & 10 & 15 & \\
\hline$\$ 120,000-\$ 149,999$ & 11 & 20 & \\
\hline$>\$ 150,000$ & 28 & 45 & \\
\hline Highest Level of Education & & & .92 \\
\hline Elementary & 1 & 2 & \\
\hline Secondary & 9 & 9 & \\
\hline College & 42 & 62 & \\
\hline University & 42 & 60 & \\
\hline Graduate School & 28 & 47 & \\
\hline Family Situation & & & .45 \\
\hline Single Parent & 22 & 23 & \\
\hline Double Parent & 94 & 153 & \\
\hline Other & 2 & 2 & \\
\hline
\end{tabular}

Frequencies $(n)$ unless otherwise noted. Groups were compared using independent $t$-tests for continuous data and $x^{2}$ tests were used for categorical

A number of physical activity interventions have transpired in childcare centres with varying success. Specifically, Bellows et al. [57], who implemented the Mighty Moves $^{\bullet}$ intervention (consisting of 15-20 min classroom lessons focused on gross motor skill acquisition for 18 weeks) found no significant effect of their intervention on preschoolers' physical activity. Similarly, Jones and colleagues [58] implemented the Jump Start 
Table 3 Means (Standard Deviations) of physical activity intensities (min/hr) for preschoolers in the control and experimental conditions

\begin{tabular}{|c|c|c|c|c|c|c|c|c|}
\hline \multirow[t]{2}{*}{ Time } & \multicolumn{2}{|l|}{ LPA } & \multicolumn{2}{|l|}{ MVPA } & \multicolumn{2}{|l|}{ TPA } & \multicolumn{2}{|c|}{ Sedentary Time } \\
\hline & Control & Exp. & Control & Exp. & Control & Exp. & Control & Exp. \\
\hline Baseline & $21.20(3.69)$ & $21.64(2.93)$ & $5.96(2.32)$ & $5.38(2.12)$ & $27.15(5.13)$ & $27.02(4.15)$ & $32.85(5.25)$ & $32.98(4.15)$ \\
\hline Post & $20.28(3.23)$ & $21.85(3.07)$ & $6.33(2.63)$ & $7.05(2.63)$ & $26.61(4.86)$ & $28.89(4.38)$ & $33.39(4.86)$ & $31.11(4.38)$ \\
\hline Diff (95\% Cl) & \multicolumn{2}{|c|}{$0.91(-0.29$ to 2.11$)$} & \multicolumn{2}{|c|}{$1.28(0.57$ to 1.99$)$} & \multicolumn{2}{|c|}{2.15 (0.58 to 3.72 ) } & \multicolumn{2}{|c|}{$-2.13(-3.72$ to -0.54$)$} \\
\hline 6 month & $20.89(3.88)$ & $20.58(5.04)$ & $5.94(2.11)$ & $4.69(1.86)$ & $26.83(4.80)$ & $25.27(6.37)$ & $33.17(4.80)$ & $34.73(6.37)$ \\
\hline Diff (95\% Cl) & \multicolumn{2}{|c|}{$-0.79(-2.30$ to 0.72$)$} & \multicolumn{2}{|c|}{$-0.58(-1.48$ to 0.32$)$} & \multicolumn{2}{|c|}{$-1.38(-3.36$ to 0.60$)$} & \multicolumn{2}{|c|}{$1.40(-0.60$ to 3.40$)$} \\
\hline 12 month & $20.11(2.74)$ & $19.93(3.73)$ & $6.25(2.16)$ & $5.96(2.57)$ & $26.36(3.68)$ & $25.90(5.63)$ & $33.64(3.68)$ & $34.10(5.63)$ \\
\hline Diff (95\% Cl) & \multicolumn{2}{|c|}{$-0.87(-2.54$ to 0.80$)$} & \multicolumn{2}{|c|}{$-0.19(0.51)(-1.19$ to 0.81$)$} & \multicolumn{2}{|c|}{$-1.04(-3.22$ to 1.14$)$} & \multicolumn{2}{|c|}{$1.16(-1.05$ to 3.37$)$} \\
\hline
\end{tabular}

LPA light physical activity, MVPA moderate-to-vigorous physical activity, TPA total physical activity, Exp. experimental condition

Reported differences for each time period represent the difference between groups (experimental minus control), when compared with baseline

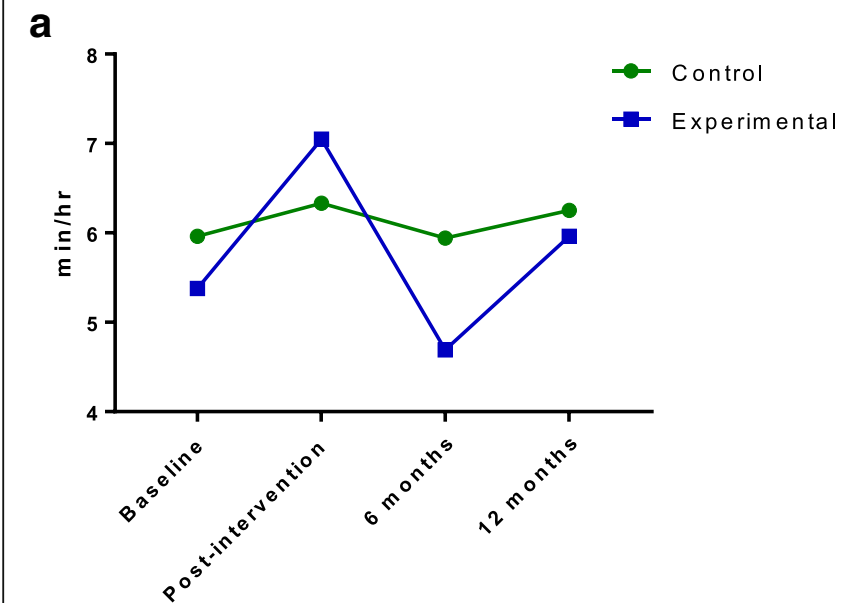

Measurement Time

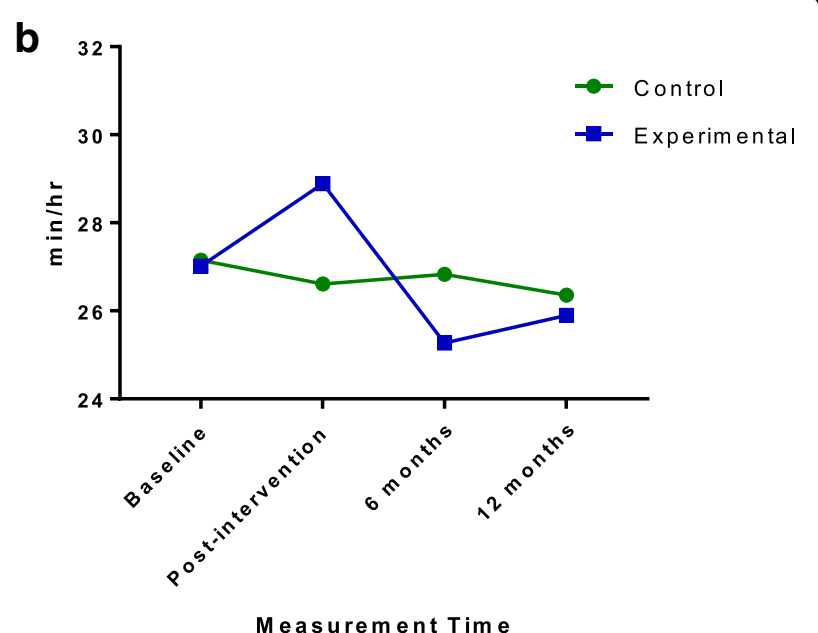

Measurement Time

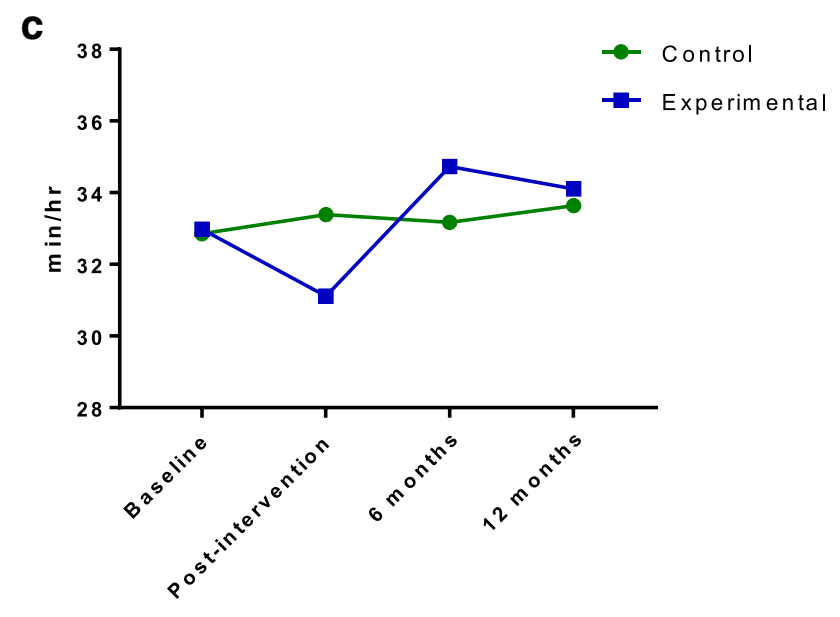

Measurement Time

Fig. 3 a, b, c Preschoolers' moderate-to-vigorous physical activity (a), total physical activity (b), and sedentary time (c) in minutes per hour across four measurement times for experimental and control groups 
Table 4 The Effect of the SPACE Intervention on LPA, MVPA, TPA, and Sedentary Time

\begin{tabular}{|c|c|c|c|c|c|c|c|c|}
\hline Model & LPA & & MVPA & & TPA & & Sedentary Time & \\
\hline${ }^{a}$ Main effects & $x^{2}(4)=13.24$ & $p=.01$ & $x^{2}(4)=43.67$ & $p<.0001$ & $x^{2}(4)=14.32$ & $p=.006$ & $x^{2}(4)=13.72$ & $p=.008$ \\
\hline${ }^{b}$ Interaction & $x^{2}(3)=6.52$ & $p=.09$ & $x^{2}(3)=19.44$ & $p=.0002$ & $x^{2}(3)=14.92$ & $p=.002$ & $x^{2}(3)=14.70$ & $p=.002$ \\
\hline
\end{tabular}

LPA light physical activity, MVPA moderate-to-vigorous physical activity, TPA total physical activity

atested against the null model

$b_{\text {tested against the main effects model }}$

program which also focused on preschoolers' gross motor skills and comprised professional development for childcare staff in addition to structured and unstructured activities for children. While the results of their intervention were not significant, improvements in activity behaviours were reported for children enrolled in the intervention. In a large Belgian population, De Creamer and colleagues found the ToyBox intervention, which transpired in Kindergarten classrooms, but included parents and families in the intervention, had a significant effect on preschoolers' activity, but noted this effect was strongest for boys and for preschoolers from higher socioeconomic classes [59]. Finally, Goldfield et al. [34] adopted a train-the-trainer approach and coupled it with a resource manual which provided childcare staff with guidance on facilitating structured and unstructured physical activities. Preschoolers in the intervention group displayed greater increases in minutes of TPA and LPA, but not MVPA at 6-months [34]. Despite the inconsistent findings reported in these 4 studies, childcare centres still maintain promise as an appropriate venue for intervening given the substantial portion of their day that young children spend in these venues [19]. Moreover, young children have been noted to accumulate more MVPA in care, compared to at home [60], which supports the feasibility of higher intensity physical activity in the childcare environment.

The SPACE intervention involved two common intervention components - environmental modifications; specifically, the addition of portable play equipment [61] as well as the provision of staff training [34]. The modification to the required outdoor playtime schedules at centre-based childcare facilities has been a less common approach to childcare interventions. To our knowledge, only a few interventions have adopted a similar approach and the results remain ambiguous. Wolfenden and colleague's [38] intervention divided the typical single 45-min bout of outdoor playtime afforded in Australian childcare centres into three shorter outdoor sessions of 15 min each; however, no results have been published to date. Similar to the SPACE intervention, Alhassan and colleagues [62] also implemented four 30-min outdoor play sessions in a small number of childcare centres in the United States. While these researchers found no significant effect of their intervention on Latino preschoolers' MVPA, it is possible this was a consequence of the short implementation timeframe of only two days.
The SPACE intervention's success at improving preschoolers' MVPA is noteworthy given the many health benefits associated with higher intensity activity, and the notion that most childcare interventions to date have been more effective at increasing lower intensity activity among this population [34]. In light of the significant (albeit short-term) changes in MVPA, TPA, and sedentary time among preschoolers in the SPACE intervention, additional research is needed to confirm the utility of a modified outdoor playtime schedule in childcare centres as a mechanism to support increased activity participation.

Researchers have previously identified outdoor playtime as a strong correlate of young children's physical activity [63, 64]. In fact, Vanderloo and colleagues [27] noted that preschoolers were two times more active outdoors than indoors, averaging $31.68 \mathrm{~min} / \mathrm{h}$ compared to $14.42 \mathrm{~min} / \mathrm{h}$ outdoors versus indoors, respectively. With consideration of higher intensity activity, these same researchers reported that preschoolers' MVPA levels were 10 times higher outdoors compared to indoors. Moreover, as noted previously, researchers have found that preschoolers display the most physical activity during the first $10 \mathrm{~min}$ outdoors $[29,65]$ and that activity is most intense during this time [30]. To capitalize on this peak in activity participation for this cohort, a modified outdoor playtime schedule was afforded to preschoolers in the SPACE intervention; specifically, four shorter outdoor sessions, compared to the typical two sessions (preschoolers' total outdoor time did not change $-2 \mathrm{~h}$ per day). Given the intermittent nature of young children's activity behaviours [66], offering more frequent, but shorter outdoor playtime sessions, appears to be a viable approach for promoting improved activity behaviours. Despite noted challenges with implementing increased outdoor playtime sessions within their current curriculum, our process evaluation, which captured attendance, adherence, dose delivered, content and feasibility, noted an adherence rate (over 70\%) to these outdoor sessions in the SPACE intervention was high (Driediger MV, Vanderloo LM, Burke SM, Irwin JD, Gaston A, Timmons BW, et al. The feasibility and appropriateness of the supporting physical activity in the childcare environment (SPACE) intervention: a process evaluation. Health Educ Behav. Submitted). This suggests that the modified playtime schedule was feasible for childcare staff to follow. 
In addition to significantly increasing physical activity levels, the SPACE intervention was also effective at decreasing preschoolers' sedentary time. This finding is similar to Goldfield et al. [34] who also displayed significant decreases in sedentary time as a consequence of their childcare-based intervention. De Craemer and colleagues [33], who implemented the ToyBox-study, a kindergarten-based, family involved, intervention in over 800 Belgian preschoolers, found no significant decreases in sedentary time among their sample. However, a decrease in objectively and subjectively measured sedentary time was observed among preschoolers from the intervention group who were enrolled in high socioeconomic status kindergartens. A great deal of attention has recently been placed on sedentary behaviours and their associated negative health consequences; therefore, the effectiveness of the SPACE intervention to decrease this behaviour is noteworthy [10]. The replacement of sedentary time with MVPA, as observed in the present study, is a promising first step.

Although the SPACE intervention was successful at increasing activity behaviours and decreasing sedentary time while the program was running, these increases were not sustained long-term. Of note, the 6-month follow-up measures were conducted during the winter months in Canada where snow and extreme cold temperatures may limit outdoor time, which may negatively impact physical activity [67, 68]. The childcare centres assigned to the intervention condition kept the portable play equipment for use post-intervention and the childcare staff received a booster session at 4 months postintervention to refresh their knowledge on the importance of physical activity for young children. As such, it seems possible that the outdoor playtime modification, more so than the provision of equipment and staff training, is the most promising for improving physical activity participation in childcare. However, this will require additional study to determine the independent effects of outdoor playtime modification.

While the present study has many strengths including its study design (i.e., clustered RCT), unique combination of evidence-informed components, and use of objective assessment tools (i.e., Actical ${ }^{\mathrm{mt}}$ accelerometers), it is not without limitations. We lost participants at follow-up (6- and 12-months) due to attrition, which occurred due to absences or withdrawals from childcare. While every effort was made to recruit participants who anticipated being in childcare for the next 12 months, the transient nature of childcare and the long study follow-up period at a very young age (with many of these children transitioning into elementary school) was problematic. Moreover, part time enrolment (e.g., half days) meant some participants were not included in the analysis due to inadequate accelerometer wear-time. Despite these limitations, this study provides insightful information about the potential of modifying outdoor playtime as a feasible approach to supporting preschoolers' physical activity.

\section{Conclusion}

The SPACE intervention was effective at improving rates of MVPA and TPA, while reducing sedentary time after the 8-week intervention; however, this effect was not sustained at 6- or 12-month follow-up. While the intervention was successful in the short-term, given the lack of long-term impact, it is possible that that modified outdoor playtime schedule, more so than the staff training and environmental modifications influenced changes in activity behaviours. Additional research is needed to determine whether a restructuring of outdoor play periods alone can produce an increase in preschoolers' physical activity levels.

\section{Abbreviations \\ CONSORT: Consolidated Standards of Reporting Trials; ISRCTN: International Standard Randomised Controlled Trial Number; LPA: Light physical activity; MVPA: moderate-to-vigorous physical activity; RCT: Randomized controlled trial; REB: Research ethics board; SPACE: Supporting Physical Activity in the Childcare Environment; TPA: Total physical activity}

\section{Acknowledgements \\ PT holds an Early Researcher Award from the Ministry of Research and Innovation. LMV was funded by the Canadian Institutes of Health Research Frederick Banting and Charles Best Doctoral Research Award. BWT holds a Canada Research Chair in Child Health and Exercise Medicine. We would like to thank the childcare staff and preschoolers for their participation in this study.}

\section{Funding}

The trial was funded by the Canadian Institutes of Health Research (CIHR grant \#133559).

\section{Availability of data and materials \\ The datasets generated and analysed during the current study is available from the corresponding author on reasonable request.}

\section{Authors' contributions}

PT, SMB, AG, JDI, AMJ, BWT, and LMV conceived and designed the study and were involved with the successful funding application for the SPACE study.

AMJ led the analyses, with assistance from PT, LMV, and BWT, and all authors assisted with the interpretation of the results. PT and LMV drafted the manuscript, and all authors provided important intellectual contributions to the final document. All authors read and approved the final manuscript.

\section{Ethics approval and consent to participate}

This study and all related documents received ethical approval from the University of Western Ontario's Research Ethics Board (REB\# 105779).

\section{Consent for publication}

Not applicable.

\section{Competing interests}

The authors declare that they have no competing interests.

\section{Publisher's Note}

Springer Nature remains neutral with regard to jurisdictional claims in published maps and institutional affiliations. 


\section{Author details}

${ }^{1}$ School of Occupational Therapy, Faculty of Health Sciences, University of Western Ontario, 1201 Western Road, Elborn College, Room 2547, London, ON N6G 1H1, Canada. ${ }^{2}$ Health and Rehabilitation Sciences, Faculty of Health Sciences, University of Western Ontario, London, ON, Canada. ${ }^{3}$ School of Health Studies, Faculty of Health Sciences, University of Western Ontario, London, ON, Canada. ${ }^{4}$ School of Kinesiology, Faculty of Health Sciences, University of Western Ontario, London, ON, Canada. ${ }^{5}$ Child Health \& Exercise Medicine Program, McMaster University, Hamilton, Canada.

\section{Received: 8 May 2017 Accepted: 29 August 2017}

\section{Published online: 07 September 2017}

\section{References}

1. Vanderloo LM, Tucker P, Johnson AM, Burke SM, Irwin JD. Environmental influences on preschoolers' physical activity levels in various early learning facilities. Res Q Exerc Sport. 2015;19:1-11.

2. Vanderloo LM, Tucker $P$, Johnson AM, Van Zandvoort MM, Burke SM, Irwin JD. The influence of centre-based childcare on preschoolers' physical activity levels: a cross-sectional study. Int J Environ Res Public Health. 2014;11:1794-802.

3. Pate RR, Pfeiffer KA, Trost SG, Ziegler P, Dowda M. Physical activity among children attending preschools. Pediatrics. 2004;114:1258-63.

4. O'Dwyer MV, Fairclough SJ, Ridgers ND, Knowles ZR, Foweather L, Stratton G. Effect of a school-based active play intervention on sedentary time and physical activity in preschool children. Health Educ Res. 2013:1-12.

5. Vanderloo LM. Screen-viewing among preschoolers in childcare: a systematic review. BMC Pediatr. 2014;14:205. doi:10.1186/1471-2431-14-205.

6. Tucker P, Vanderloo LM, Burke SM, Irwin JD, Johnson AM. Prevalence and influences of preschoolers' sedentary behaviors in early learning centers: a cross-sectional study. BMC Pediatr. 2015;15:128. doi:10.1186/s12887-015-0441-5.

7. Ellis YG, Cliff DP, Janssen X, Jones RA, Reilly JJ, Okely AD. Sedentary time, physical activity and compliance with IOM recommendations in young children at childcare. Preventive Medicine Reports. 2017. doi.org/10.1016/j. pmedr.2016.12.009.

8. Timmons BW, Leblanc AG, Carson V, Gorber SC, Dillman C, Janssen I, et al. Systematic review of physical activity and health in the early years (aged 0-4 years). Appl Physiol Nutr Metab. 2012;37:773-92.

9. Timmons, Naylor P, Pfeiffer KA. Physical activity for preschool children-how much and how? Can J Public Health. 2007;98(Suppl 2):S122-34.

10. Leblanc AG, Spence JC, Carson V, Connor Gorber S, Dillman C, Janssen I, et al. Systematic review of sedentary behaviour and health indicators in the early years (aged 0-4 years). Appl Physiol Nutr Metab. 2012;37:753-72.

11. Carson V, Hunter S, Kuzik N, Wiebe SA, Spence JC, Friedman A, et al. Systematic review of physical activity and cognitive development in early childhood. J Sci Med Sport. 2016;19:573-8.

12. Canadian Society of Exercise Physiology. Canadian physical activity guidelines for the early years: 0-4 years. 2012. http://www.csep.ca/cmfiles/ guidelines/csep_paguidelines early-years en.pdf. Accessed May 7, 2017

13. Canadian Society of Exercise Physiology. Canadian sedentary behaviour guidelines for the early years (0-4 years). 2012. http://www.csep.ca/CMFiles/ Guidelines/CSEP_SBGuidelines_early-years_en.pdf. Accessed May 7, 2017.

14. Benjamin SE, Cradock A, Walker EM, Slining M, Gillman MW. Obesity prevention in child care: a review of U.S. state regulations. BMC Public Health. 2008:8:188.

15. Hinkley T, Salmon J, Okely AD, Crawford D, Hesketh K. Preschoolers' physical activity, screen time, and compliance with recommendations. Med Sci Sports Exerc. 2012;44:458-65.

16. Ward D, Hales D, Haverly K, Marks J, Benjamin S, Ball S, et al. An instrument to assess the obsogenic environment of child care centers. Am J Health Behav. 2008;32:380-6.

17. Finn K, Johannsen N, Specker B. Factors associated with physical activity in preschool children. J Pediatr. 2002;140:81-5.

18. Cleveland G, Forer B, Hyatt D, Japel C, Krashinsky M. New evidence about child care in Canada: use patterns, affordability and quality. Inst Res Public Policies. 2008;14:1-44.

19. Sinha M. Spotlight on Canadians: results from the general social survey: child care in Canada. 2014. http://www.statcan.gc.ca/pub/89-652-x/89-652x2014005-eng.htm. Accessed May 7, 2017.

20. Geoffroy MC, Power C, Touchette E, Dubois L, Boivin M, Seguin JR, et al. Childcare and overweight or obesity over 10 years of follow-up. J Pediatr. 2012;162:753-8.
21. Ward DS, Vaughn A, McWilliams C, Hales D. Physical activity at child care settings: review and research recommendations. Am J Lifestyle Med. 2009;3:474-88.

22. Gordon ES, Tucker P, Burke SM, Carron AV. Effectiveness of physical activity interventions for preschoolers: a meta-analysis. Res Q Exerc Sport. 2013:84:287-94.

23. Baranowski T, Thompson WO, Durant RH, Baranowski J, Puhl J. Observations on physical activity in physical locations: age, gender, ethnicity, and month effects. Res Q Exerc Sport. 1993;64:127-33.

24. Cosco NG, Moore RC, Islam MZ. Behavior mapping: a method for linking preschool physical activity and outdoor design. Med Sci Sports Exerc. 2010; 42:513-9.

25. Hinkley T, Crawford D, Salmon J, Okely AD, Hesketh K. Preschool children and physical activity: a review of correlates. Am J Prev Med. 2008;34:435-41.

26. Alhassan S, Nwaokelemeh O, Mendoza A, Shitole S, Whitt-Glover M, Yancey A. Design and baseline characteristics of the short bouTs of exercise for preschoolers (STEP) study. BMC Public Health. 2012;12:582.

27. Vanderloo LM, Tucker P, Johnson AM, Holmes JD. Physical activity among preschoolers during indoor and outdoor childcare play periods. Appl Physiol Nutr Metab. 2013;38:1173-5.

28. Tremblay SM, Gray C, Babcock S, Barnes J, Bradstreet CC, Carr D et al. Position Statement on Active Outdoor Play 2015, 12. https://www. participaction.com/sites/default/files/downloads/ParticipactionPositionStatement-ActiveOutdoorPlay_0.pdf. Accessed May 7, 2017.

29. Pate RR, Dowda M, Brown WH, Mitchell J, Addy C. Physical activity in preschool children with the transition to outdoors. J Physl Act Health. 2013;10:170-5.

30. Greever CJ, Sirard J, Alhassan S. Objective analysis of preschoolers' physical activity patterns during free playtime. J Physl Act Health. 2015;12:1253-8.

31. Pate RR, Brown WH, Pfeiffer KA, Howie EK, Saunders RP, Addy CL, et al. An intervention to increase physical activity in children: a randomized controlled trial with 4-year-olds in preschools. Am J Prev Med. 2016;51:12-22.

32. De Bock F, Genser B, Raat H, Fischer JE, Renz-Polster HA. Participatory physical activity intervention in preschools: a cluster randomized controlled trial. Am J Prev Med. 2013;45:64-74.

33. De Craemer M, De Decker E, Verloigne M, De Bourdeaudhuij I, Manios Y, Cardon G. The effect of a cluster randomised control trial on objectively measured sedentary time and parental reports of time spent in sedentary activities in Belgian preschoolers: the ToyBox-study. Int J Behav Nutr Phys Act. 2016;13:1. doi:10.1186/s12966-015-0325-y.

34. Goldfield GS, Harvey ALJ, Grattan KP, Temple V, Naylor PJ, Alberga AS, et al. Effects of a child care intervention on physical activity and body composition. Am J Prev Med. 2016;51:225-31.

35. Alhassan S, Nwaokelemeh O, Mendoza A, Shitole S, Puleo E, Pfeiffer KA, et al. Feasibility and effects of short activity breaks for increasing preschoolage children's physical activity levels. J School Health. 2016;86:526-33. doi:10.1111/josh.12403.

36. Jones RA, Okely AD, Hinkley T, Batterham M, Burke C. Promoting gross motor skills and physical activity in childcare: a translational randomized control trial. J Sci Med Sport. 2016:1-6. doi:10.1016/j.jsams.2015.10.006.

37. Barber S, Akhtar S, Jackson C, Bingham D, Hewitt C, Routen A, et al. Preschoolers in the playground: a pilot cluster randomised controlled trial of a physical activity intervention for children aged 18 months to 4 years. Public Health Res. 2015:3.

38. Wolfenden L, Wiggers J, Morgan P, Razak LA, Jones J, Finch M, et al. A randomised controlled trial of multiple periods of outdoor free-play to increase moderate-to-vigorous physical activity among 3 to 6 year old children attending childcare: study protocol. BMC Public Health. 2016;16: 926. doi:10.1186/s12889-016-3604-X.

39. Bélanger $M$, Humbert L, Vatanparast $H$, Ward S, Muhajarine $N$, Chow AF, et al. A multilevel intervention to increase physical activity and improve healthy eating and physical literacy among young children (ages 3-5) attending early childcare centres: the healthy start-Départ Santé cluster randomised controlled trial study protocol. BMC Public Health. 2016;16:313. doi:10.1186/ s12889-016-2973-5.

40. Finch M, Wolfenden L, Morgan PJ, Freund M, Jones J, Wiggers JA. Cluster randomized trial of a multi-level intervention, delivered by service staff, to increase physical activity of children attending center-based childcare. Prev Med. 2014:58:9-16. doi:10.1016/j.ypmed.2013.10.004

41. Tucker P, Van Zandvoort MM, Burke SM, Irwin JD. Physical activity at daycare: childcare providers' perspectives for improvements. J Early Child Res. 2011;9:207-19. 
42. van Zandvoort M, Tucker P, Irwin JD, Burke SM. Physical activity at daycare: issues, challenges and perspectives. Early Years. 2010;30:175-88.

43. Tucker P, Burke SM, Gaston A, Irwin JD, Johnson AM, Timmons BW, et al. Supporting physical activity in the childcare environment (SPACE): rationale and study protocol for a cluster randomized controlled trial. BMC Public Health. 2016;16:112. doi:10.1186/s12889-016-2775-9.

44. Green LW, Kreuter MW. Health program planning: An educational and ecological approach. 4th ed. New York: New York: McGraw-hill High Educ; 2005

45. Campbell MK, Piaggio G, Elbourne DR, Altman DG. For the CONSORT group. Consort 2010 statement: extension to cluster randomised trials. BMJ. 2012; 345:5661.

46. Cliff DP, Reilly JJ, Okely AD. Methodological considerations in using accelerometers to assess habitual physical activity in children aged 0-5 years. J Sci Med Sport. 2009;12:557-67.

47. Pate RR, Almeida MJ, Mclver KL, Pfeiffer KA, Dowda M. Validation and calibration of an accelerometer in preschool children. Obesity. 2006;14 2000-2006

48. Colley R, Garriguet D, Adamo K, Carson V, Janssen I, Timmons B, et al. Physical activity and sedentary behavior during the early years in Canada: a cross-sectional study. Int J Behav Nutr Phys Act. 2013;10:54.

49. Adolph AL, Puyau MR, Vohra FA, Nicklas TA, Zakeri IF, Butte NF. Validation of uniaxial and triaxial accelerometers for the assessment of physical activity in preschool children. J Physl Act Health. 2012;9:944-53.

50. Wong SL, Colley RC, Connor Gorber S, Tremblay MS. Actical accelerometer sedentary activity thresholds for adults. J Physl Act Health. 2011:8:587-91.

51. Carson V, Clark D, Ogden N, Harber V, Kuzik N. Short-term influence of revised provincial accreditation standards on physical activity, sedentary behavior, and weight status in Alberta, Canada child care centers. Early Child Educ J. 2015;43(6):459-65. doi:10.1007/s10643-015-0688-3.

52. Kuzik N, Clark D, Ogden N, Harber V, Carson V. Physical activity and sedentary behaviours of toddlers and preschoolers in child care centres in Alberta, Canada. Can J Public Health. 2015;106:e178-83. doi: 10.17269/CJPH. 106.4794 .

53. Schaalje GB, McBride JB, Fellingham GW. Adequacy of approximations to distributions of test statistics in complex mixed linear models. J Agric Biol Environ Stat. 2002;7:512.

54. Bates D, Machler M, Bolker B, Walker S. Fitting linear mixed-effects models using Ime4. J Stat Softw. 2015;67:1-48.

55. R Core Team. R. A language and environment for statistical computing. Vienna, Austria: R Foundation for Statistical Computing; 2017.

56. Kuznetsova A, Brockhoff PB, Bojesen Christensen RH. ImerTest: tests in linear mixed effects models. [R package version 2.0-33]. 2016.

57. Bellows L, Davies PL, Anderson J, Kennedy C. Effectiveness of a physical activity intervention for head start preschoolers: a randomized intervention study. Am J Occup Ther 2013;67:28-36. doi: 10.5014.ajot.2013.005777.

58. Jones RA, Okely AD, Hinkley T, Batterham M, Burke C. Promoting gross motor skills and physical acntivity in childcare: a tranlational randomized controlled trial. J Sci Med Sport. 2015;19(9):744-9. doi:10.1016/j.jsams.2015.10.006.

59. De Craemer M, De Decker E, Verloigne M, De Bourdeaudhuij I, Manios Y, Cardon, G. The effect of a kindergarten-based, family-involved intervention on objectively measured physical activity in Belgian preschool boys and girls of high and low SES: the ToyBox-study. Int J Behav Nutri Phys Act 2014;11:38.

60. Hesketh KR, Griffin SJ, van Sluijs EMFUK. Preschool-aged children's physical activity levels in childcare and at home: a cross-sectional exploration. Int J Behav Nutr Phys Act. 2015;12:123. doi:10.1186/s12966-015-0286-1.

61. Bower JK, Hales DP, Tate DF, Rubin DA, Benjamin SE, Ward DS. The childcare environment and children's physical activity. Am J Prev Med. 2008;34:23-9.

62. Alhassan S, Sirars JR, Robinson TN. The effects of increasing outdoor play time on physical activity in Latino preschool children. Int J Pediatr Obes. 2007;2:153-8.

63. Henderson KE, Grode GM, O'Connell ML, Schwartz MB. Environmental factors associated with physical activity in childcare centers. Int J Behav Nutr Phys Act. 2015;12:1-9. doi:10.1186/s12966-015-0198-0.

64. Schmutz EA, Leeger-Aschmann CS, Radtke T, Muff S, Kakebeeke TH, Zysset $A E$, et al. Correlates of preschool childrens' objectively measured physical activity and sedentary behavior: a cross-sectional analysis of the SPLASHY study. Int J Behav Nutr Phys Act. 2017;14:1. doi: 10.1186/s12966-016-0456-9 .

65. McKenzie TL, Sallis JF, Elder JP, Berry CC, Hoy PL, Nader PR, et al. Physical activity levels and prompts in young children at recess: a two-year study of a bi-ethnic sample. Res Q Exerc Sport. 1997;68:195-202.
66. Benham-Deal T. Preschool children's accumulated and sustained physical activity. Percept Mot Skills. 2005;100:443-50.

67. Carson V, Spence JC. Seasonal variation in physical activity among children and adolescents: a review. Pediatr Exerc Sci. 2010;22(1):81-92.

68. Tucker P, Gilliland J. The effect of season and weather on physical activity: a systematic review. Public Health. 2007;121(12):909-22.

\section{Submit your next manuscript to BioMed Central and we will help you at every step:}

- We accept pre-submission inquiries

- Our selector tool helps you to find the most relevant journal

- We provide round the clock customer support

- Convenient online submission

- Thorough peer review

- Inclusion in PubMed and all major indexing services

- Maximum visibility for your research

Submit your manuscript at www.biomedcentral.com/submit
Ciomed Central 